\title{
SwarmPlay: A Swarm of Nano-Quadcopters Playing Tic-tac-toe Board Game against a Human
}

\author{
Ekaterina Karmanova \\ Skolkovo Institute of Science and \\ Technology \\ Moscow, Russia \\ ekaterina.karmanova@skoltech.ru

\section{Roman Ibrahimov} \\ Skolkovo Institute of Science and \\ Technology \\ Moscow, Russia \\ roman.ibrahimov@skoltech.ru
}

\author{
Valerii Serpiva \\ Skolkovo Institute of Science and \\ Technology \\ Moscow, Russia \\ valerii.serpiva@skoltech.ru
}

\section{Aleksey Fedoseev}

Skolkovo Institute of Science and

Technology

Moscow, Russia

aleksey.fedoseev@skoltech.ru

\author{
Stepan Perminov \\ Skolkovo Institute of Science and \\ Technology \\ Moscow, Russia \\ stepan.perminov@skoltech.ru
}

Dzmitry Tsetserukou

Skolkovo Institute of Science and

Technology

Moscow, Russia

d.tsetserukou@skoltech.ru

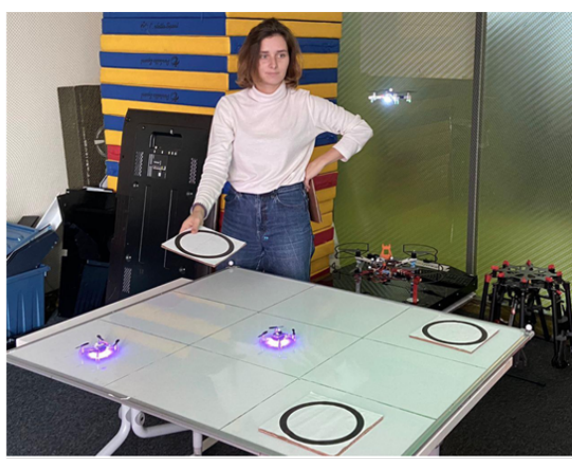

a)

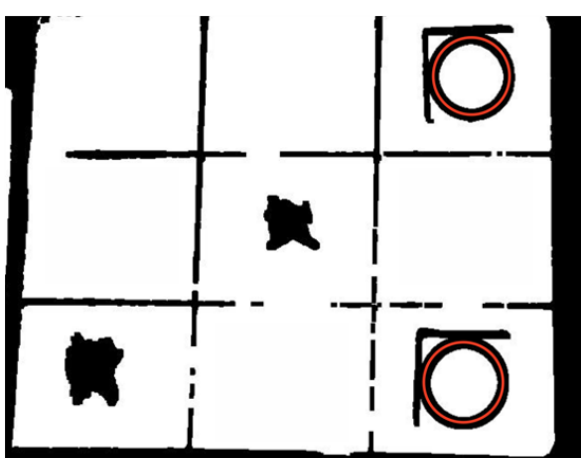

b)

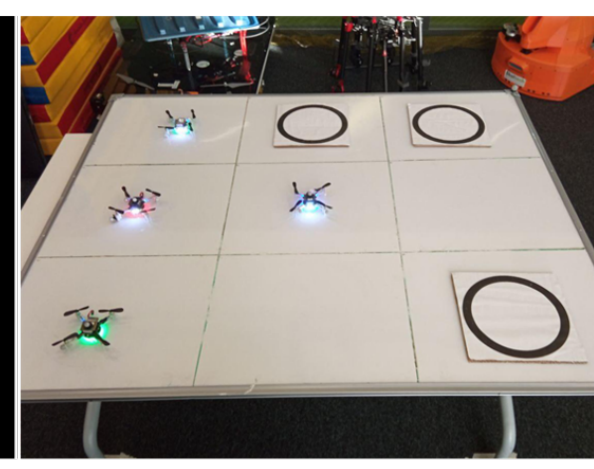

c)

Figure 1: Tic-tac-toe game with the swarm of drones: a) A human plays Tic-tac-toe board game against a SwarmPlay. b) Game board image transformed by the $\mathrm{CV}$ system. c) A closed view on the board when drones won the match.

\begin{abstract}
We present a new paradigm of games, i.e. SwarmPlay, where each playing component is presented by an individual drone that has its own mobility and swarm intelligence to win against a human player. The motivation behind the research is to make the games with machines tangible and interactive. Although some research on the robotic players for board games already exists, e.g., chess, the SwarmPlay technology has the potential to offer much more engagement and interaction with a human as it proposes a multi-agent swarm instead of a single interactive robot. The proposed system consists of a robotic swarm, a workstation, a computer vision (CV), and Game Theory-based algorithms. A novel game algorithm was developed to provide a natural game experience to the user. The

Permission to make digital or hard copies of all or part of this work for personal or classroom use is granted without fee provided that copies are not made or distributed for profit or commercial advantage and that copies bear this notice and the full citation on the first page. Copyrights for components of this work owned by others than ACM must be honored. Abstracting with credit is permitted. To copy otherwise, or republish, to post on servers or to redistribute to lists, requires prior specific permission and/or a fee. Request permissions from permissions@acm.org.

SIGGRAPH '21 Emerging Technologies, August 9-13, 2021, Virtual Event, USA

(C) 2021 Association for Computing Machinery.

ACM ISBN 978-1-4503-8364-6/21/08 . .\$15.00

https://doi.org/10.1145/3450550.3465346
\end{abstract}

preliminary user study revealed that participants were highly engaged in the game with drones $(69 \%$ put a maximum score on the Likert scale) and found it less artificial compared to the regular computer-based systems ( $77 \%$ put maximum score). The affection of the user's game perception from its outcome was analyzed and put under discussion. User study revealed that SwarmPlay has the potential to be implemented in a wider range of games, significantly improving human-drone interactivity.

\section{CCS CONCEPTS}

- Human-centered computing $\rightarrow$ Human-computer interaction (HCI).

\section{KEYWORDS}

Human-Drone Interaction (HDI), Game Theory, Computer Vision, Multi-Agent Systems

\section{ACM Reference Format:}

Ekaterina Karmanova, Valerii Serpiva, Stepan Perminov, Roman Ibrahimov, Aleksey Fedoseev, and Dzmitry Tsetserukou. 2021. SwarmPlay: A Swarm of Nano-Quadcopters Playing Tic-tac-toe Board Game against a Human. In Special Interest Group on Computer Graphics and Interactive Techniques Conference Emerging Technologies (SIGGRAPH '21 Emerging Technologies ), 
August 9-13, 2021, Virtual Event, USA. ACM, New York, NY, USA, 4 pages. https://doi.org/10.1145/3450550.3465346

\section{INTRODUCTION}

One of the potential domains for human-robot interaction research is physical board games that have an adjustable structure level. Perceiving the game components and the board, understanding human movements, reasoning about the state, and manipulating the game components to win against human players are integral steps in robot-centric board games [Li et al. 2019; Matuszek et al. 2011].

For the human player, on the other side, the interaction with a robot provides a fresh perspective on the well-known competitive games, e.g., robotic rock-paper-scissors with humanoid robot RASA presented in [Ahmadi et al. 2019]. Nowadays much work is aimed to improve the AI in such games, either by more sufficient game strategy systems, e.g., gaming decision system developed for Go in [Silver et al. 2016] and curling robot with adaptive deep reinforcement learning framework proposed in [Won et al. 2020], or by improving the estimation of the human behavior, e.g., tennis player's movement prediction proposed in [Wu and Koike 2020]. However, the system architecture in robot-centric applications has been relatively little investigated and is narrowed to the single robotic manipulators and mobile robots [Becker-Asano et al. 2014; Kyohei et al. 2020; Nugroho et al. 2014]. The research on multi-robot games though is mostly focused on coordination between robotic agents, such as soccer game strategies suggested in [Reis et al. 2013] and [Liu 2020] that exclude human from the gaming stage.

To upgrade the level of engagement and interactivity of traditional games, we suggest a novel game paradigm where each game piece has its own mobility, and behaves jointly with other agents to win against the opponent. The proposed SwarmPlay technology provides CV-driven Human-Swarm interaction (HSI) in board games. To our knowledge, our prototype is the first approach towards using a multi-UAV system in physical games that involves human presence. This research focuses on the system architecture and its validation by user study, followed by a discussion about future work and potential SwarmPlay game applications.

\section{SYSTEM OVERVIEW}

\subsection{System Architecture}

The developed SwarmPlay system consists of Vicon Tracking system with 12 IR cameras for drone localization, a CV camera for game state evaluation, a drone landing table with a game board, PC with Mocap framework and PC with a drone-control framework, a CV system, and a decision-making system (Fig. 2). A white board was divided into 9 cells according to Tic-tac-toe rules. According to the specified algorithm, drones were landing on a board's cells representing Crosses (Xs). Whereas a human plays Noughts (Ox), placing cards with printed circles on the white board.

To obtain pictures of the game board providing awareness of a current status of the game, we used a camera Logitech HD Pro Webcam C920 of @30FPS mounted on the ceiling of the room. The game board is placed right under the camera. The pictures are sent to the CV system to determine the human's turn. After that, data on the human's turn as a cell number is sent to the decision-making system to define a cell where the drones should make their next turn. CV and decision-making processing is performed on Intel ${ }^{\circledR}$ Core $^{\mathrm{TM}} \mathrm{i} 7-9750 \mathrm{HF}$ CPU @ $2.60 \mathrm{GHz} \times 12$. The most recent cell data is sent to a drone-control framework. The framework obtains both the target cell, where a drone should be sent, and data from the motion capture system about current drone positions. To get the high-quality tracking of the drones, we used Vicon motion capture system with 12 cameras (Vantage V5) covering a $5 \mathrm{~m}^{3}$ space. Drones are sent to the target cells with PID control parameters, i.e., the target position, speed, and acceleration.

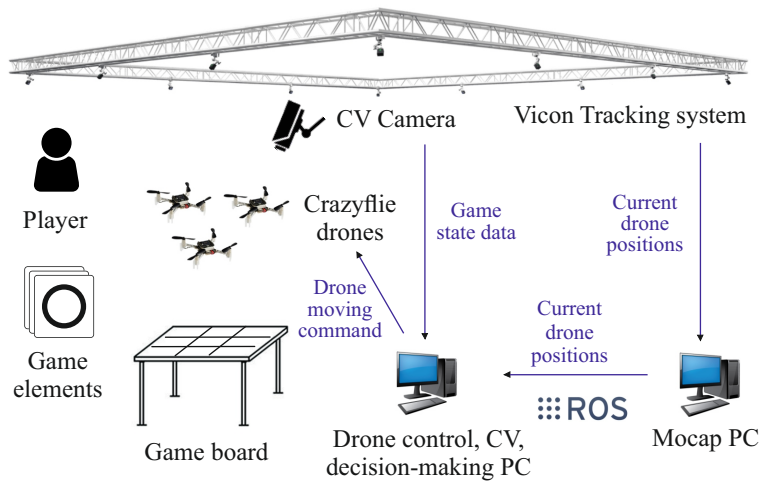

Figure 2: SwarmPlay system layout: hardware architecture.

\subsection{Computer Vision}

To detect to which cell the user puts circles, we developed a corresponding Computer Vision system. As its input, we used a picture taken by an RGB camera mounted on the ceiling of the room where the gaming board is located. At each step, the system takes a picture throughout the camera and converts it to grayscale. Then, simple thresholding and erosion with a kernel $5 \times 5$ are applied. After that, the picture is cropped and divided into 9 small images, one per a game cell. For each small image, a contour search is performed. When users make their turn, they put a circle on a cell, which is then detected as a contour by the CV system and filled with black pixels.

At the end of each step, the CV system computes the density of black pixels per a game cell. In this case, big coloured circles show a great density value. Thus, using some threshold, it makes possible to separate holes, drones, and empty areas from each other.

After detecting a new circle on the playing field, the CV system sends a corresponding game cell number, as the latest human turn, to a decision-making system to solve how exactly drones should behave in the situation.

\section{GAME STRATEGY}

\subsection{Implementation}

Tic-tac-toe game is played on a three-by-three grid. Each player takes a turn to place a symbol on an open square. The drones play as an " $\mathrm{X}$ " player, and the user is playing as an "O" player. The game is over if one of the players has three identical elements in a row: horizontally, vertically, or diagonally. The game can end with a draw 
result if there is no possibility to achieve any winning combination. The board is represented by two-dimensional matrix $3 \times 3$ (Fig. 3), where each cell was enumerated as $1,2,3, \ldots 9$. Each element of the matrix equals one of the following value: 0 : Unoccupied Cell; +1 : Drone Symbol "X"; -1: Player symbol "O".

\begin{tabular}{l|l|l}
1 & 2 & 3 \\
\hline 4 & 5 & 6 \\
\hline 7 & 8 & 9
\end{tabular}

\begin{tabular}{c|c|c}
\begin{tabular}{c|c} 
"X" \\
$(+1)$
\end{tabular} & 0 & 0 \\
\hline "X" & "O" & $\begin{array}{c}\text { "O" } \\
(+1)\end{array}$ \\
$(-1)$ & $(-1)$ \\
\hline 0 & 0 & $\begin{array}{c}\text { "X" } \\
(+1)\end{array}$
\end{tabular}

Figure 3: Game board representation in the SwarmPlay system.

\subsection{Improved Basic Algorithm}

We can choose who makes the first turn in our game: the human or the drone swarm. The algorithm with a moderate level of difficulty is implemented, which allows the human player to win/lose equally. Our developed algorithm for the Tic-tac-toe is based on the Basic Algorithm strategy introduced in [Karamchandani et al. 2015], which is focused on the computer-based game scenario.

Since the proposed drone-based scenario of Tic-tac-toe requires more preparation time and complex actions from the swarm, in this research we hypothesized that the less complexity of the game would not meet the player's expectations. To provide a considerable challenge for the user, we propose an Improved Simple Algorithm (see Algorithm 1).

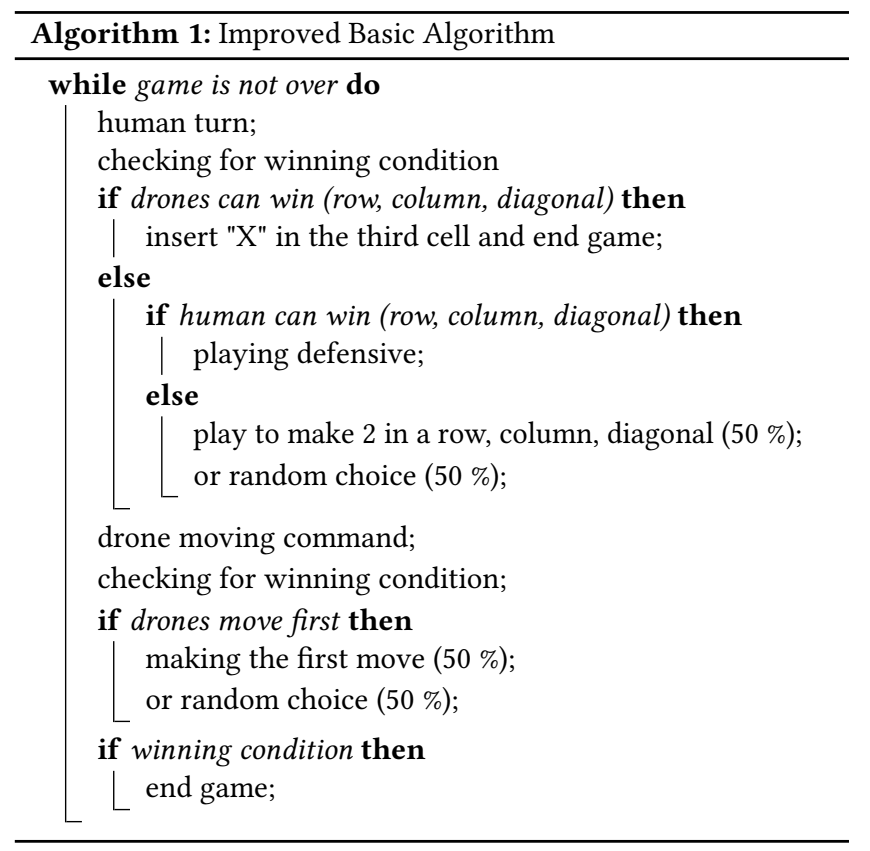

\section{EXPERIMENTS}

\subsection{Research Methodology}

Participants. We invited 13 participants aged 22 to 43 years (mean=25.6, std=5.9) to complete the survey. $15.4 \%$ of them have never interacted with drones before, $15.4 \%$ regularly deal with drones, and $69.2 \%$ periodically participate in drone-based scenarios.

Procedure. At the beginning of the experiment, the procedure and game equipment were introduced to each participant. Rules of Tic-tac-toe were described for 2 participants (15\%) who have never played the game before. The goal of Tic-tac-toe game is to make a line of 3 playing elements sooner than your opponent. Game elements, i.e., noughts for human-player and crosses for SwarmPlay, were represented by cardboard plates with printed black circles and cross shapes of the drones, respectively. Players placed the playing elements on the horizontally arranged game board, $1 \times 1.2 \mathrm{~m}$ white board with grid lines. All participants had played two matches with SwarmPlay. A human made the first move in the first game and drones in the second. After each game, the game duration and score were recorded.

At the end of the game, all respondents were asked to evaluate the SwarmPlay game with a Likert scale (1-5) on seven parameters: excitement, engagement, latency, challenge, tiredness, stress factor, and Turing test.

\subsection{Experimental results}

We conducted a chi-square analysis based on the frequency of answers in each category.

The results showed that the game parameters are all independent ( $\min p=0.14>0.05$ ). Additionally the chi-square test of independence revealed that the participants' experience with drones does not affect the evaluation of drone swarm perception criteria, such as tiredness $\left(\tilde{\chi}^{2}=10.77, p=0.29\right)$, stress factor $\left(\tilde{\chi}^{2}=2.53, p=0.87\right)$ and Turing test $\left(\tilde{\chi}^{2}=12.19, p=0.20\right)$. The results of the study are presented in Fig. 4a.

In summary, $38.5 \%$ of the participants found the game more exciting than regular paper-based game; $76.9 \%$ did not feel any discomfort playing along with drones and $69.2 \%$ of users found the SwarmPlay response fast enough $(\geq 4)$ compared to the usual human-opponent move. The results revealed that participants were fully engaged in the game based on the Improved Simple Algorithm (69\% put 5 scores, $31 \%$ - 4 scores), and $85 \%$ claimed they did not get tired playing with drones $(\leq 2)$. The proposed algorithm proved itself comparable to average person skills, allowing its opponent to win or lose equally. Only 23\% of participants considered that playing with a robotic opponent was much distinguishable from the real person. $62 \%$ of respondents evaluated the game being challenging $(\geq 4)$. Participants show a great interest to try other well-known board games in a new interpretation with a swarm of drones (Fig. 4b).

According to the results of the survey, the most popular games which people are willing to play with drones are Billiard with $20.6 \%$ user choice, followed by Battleship (17.6\%) and Tetris (17.6\%). As the results from 26 games in total, SwarmPlay managed to win $23.1 \%$ of all matches versus $26.9 \%$ of the user wins. The average duration of one game was $67.2 \mathrm{sec}$. For the games that started with 


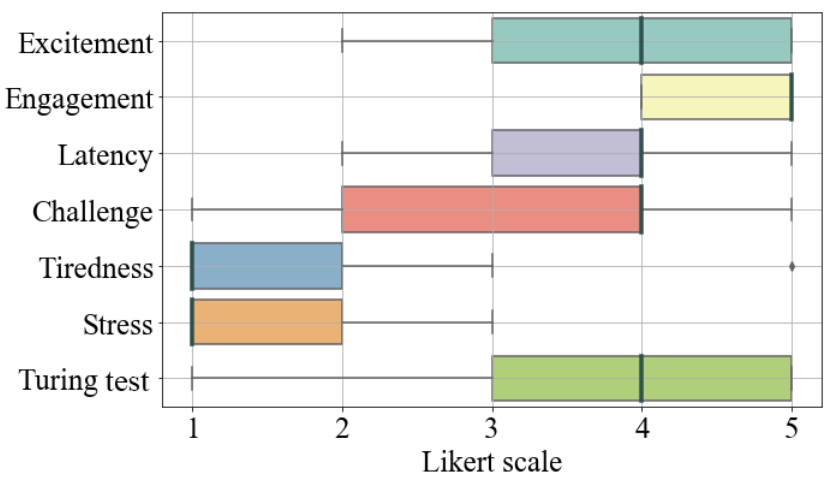

(a)

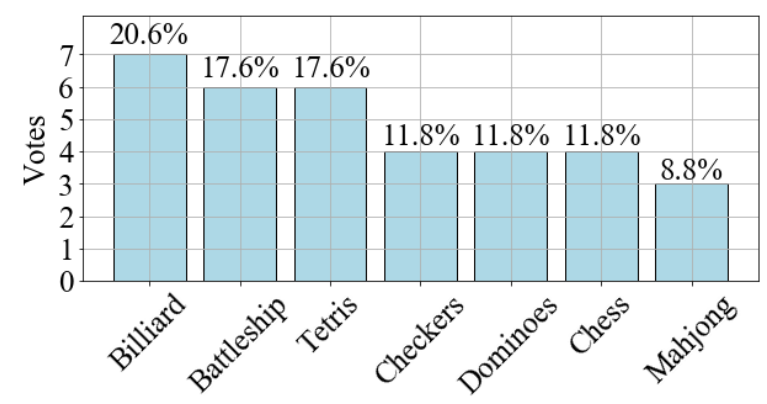

(b)

Figure 4: Experimental results: a) Subjective feedback on 5-point Likert scale. b) Results of the survey: "What other games, based on SwarmPlay, would you like to play?"

Table 1: Relation between first move and game results

\begin{tabular}{|l|c|c|c|}
\hline Result: \Start: & $\begin{array}{c}\text { SwarmPlay } \\
\text { First move }\end{array}$ & $\begin{array}{c}\text { Human } \\
\text { First move }\end{array}$ & $\begin{array}{c}\text { Average time } \\
(\mathrm{sec})\end{array}$ \\
\hline SwarmPlay won & 5 & 1 & 65.1 \\
\hline Draw & 8 & 5 & 69.7 \\
\hline Human won & 0 & 7 & 58.6 \\
\hline Games in total: & $\mathbf{1 3}$ & $\mathbf{1 3}$ & $\mathbf{6 7 . 2}$ \\
\hline
\end{tabular}

the SwarmPlay's move, the average time was $72.5 \mathrm{sec}$, which is $14.6 \%$ longer than for the games with a human's first move (61.9 sec).

Discussion. The results revealed that the first move is essential for the game outcome (see Table 1), with the first player winning in $46 \%$ cases. According to the received data, SwarmPlay won $38.5 \%$ of matches when drones started the game, while drones won only $7.7 \%$ when a human player moved first. Draw outcomes occurred more frequently when SwarmPlay started (61.5\%), than when a human started the game (38.5\%). When human players started, they won in $53.8 \%$ games.

Additionally, we discovered that the more sophisticated strategy SwarmPlay performed and the more points it had, the more humanlike behaviour of the SwarmPlay participants mentioned.

\section{CONCLUSIONS AND FUTURE WORK}

We have developed SwarmPlay, the system in which human plays against the swarm of drones. Our experimental results show that $38.5 \%$ of the participants found the game more exciting than regular paper-based games, and $76.9 \%$ of users did not feel stress while participating in the HDI scenario.

Participants were engaged with the novel drone interaction technology (engagement mean score equals 4.7 out of 5.0) and indicated their readiness to play other drone-based games such as Billiard (20.6\% user choice), Battleship (17.6\%), and Tetris (17.6\%). Therefore, the proposed SwarmPlay technology can potentially improve our way of interaction with game pieces. Machines can learn from a human's winning strategy and, more importantly, teach humans how to achieve such a strategy throughout the interaction with an intelligent swarm.

The future work will be devoted to more advanced board games, and we plan to apply ML techniques to learn the level of the player and adjust the difficulty level of the game in real-time.

\section{REFERENCES}

Ehsan Ahmadi, Ali Pour, Alireza Siamy, Alireza Taheri, and Ali Meghdari. 2019. Playing Rock-Paper-Scissors with RASA: A Case Study on Intention Prediction in Human-Robot Interactive Games. 347-357. https://doi.org/10.1007/978-3-030-35888-4 32

Christian Becker-Asano, Eduardo Meneses, Nicolas Riesterer, Julien Hué, Christian Dornhege, and Bernhard Nebel. 2014. The Hybrid Agent MARCO: A Multimodal Autonomous Robotic Chess Opponent. In Proceedings of the Second International Conference on Human-Agent Interaction (Tsukuba, Japan) (HAI '14). Association for Computing Machinery, New York, NY, USA, 173-176. https://doi.org/10.1145/ 2658861.2658915

Sunil Karamchandani, Part Gandhi, Omkar Pawar, and Shruti Pawaskar. 2015. A simple algorithm for designing an artificial intelligence based Tic Tac Toe game. In 2015 International Conference on Pervasive Computing (ICPC). 1-4. https://doi.org/10. 1109/PERVASIVE.2015.7087182

Asai Kyohei, Nakayama Masamune, and Yase Satoshi. 2020. The Ping Pong Robot to Return a Ball Precisely. In Omron TECHNICS, Vol. 51.016. 1-6. https://www.omron. com/global/en/technology/omrontechnics/vol51/016.html

Yanan Li, Gerolamo Carboni, Franck Gonzalez, Domenico Campolo, and Etienne Burdet. 2019. Differential game theory for versatile physical interaction. Nature Machine Intelligence 1 (01 2019), 36-43. https://doi.org/10.1038/s42256-018-0010-3

Xiaoli Liu. 2020. Research on decision-making strategy of soccer robot based on multiagent reinforcement learning. International fournal of Advanced Robotic Systems 17 (05 2020), 172988142091696. https://doi.org/10.1177/1729881420916960

Cynthia Matuszek, Brian Mayton, Roberto Aimi, Marc Peter Deisenroth, Liefeng Bo, Robert Chu, Mike Kung, Louis LeGrand, Joshua R Smith, and Dieter Fox. 2011. Gambit: An autonomous chess-playing robotic system. In 2011 IEEE International Conference on Robotics and Automation. IEEE, 4291-4297.

Sebastian Adi Nugroho, Ary Setijadi Prihatmanto, and Arief Syaichu Rohman. 2014. Design and implementation of kinematics model and trajectory planning for NAO humanoid robot in a tic-tac-toe board game. In 2014 IEEE 4th International Conference on System Engineering and Technology (ICSET), Vol. 4. IEEE, 1-7.

Luís Paulo Reis, Fernando Almeida, Luís Mota, and Nuno Lau. 2013. Coordination in Multi-robot Systems: Applications in Robotic Soccer. In Agents and Artificial Intelligence, Joaquim Filipe and Ana Fred (Eds.). Springer Berlin Heidelberg, Berlin, Heidelberg, 3-21.

David Silver, Ajaand Huang, Chris J. Maddison, Arthur Guez, Laurent Sifre, George van den Driessche, Julian Schrittwieser, Ioannis Antonoglou, Vedavyas Panneershelvam, Marc Lanctot, Sander Dieleman, Dominik Grewe, John Nham, Nal Kalchbrenner, Ilya Sutskever, Timothy Lillicrap, Madeleine Leach, Koray Kavukcuoglu, Thore Graepel, and Demis Hassabis. 2016. Mastering the game of Go with deep neural networks and tree search. Nature 529 (2016), 484-489.

Dong-Ok Won, Klaus-Robert Müller, and Seong-Whan Lee. 2020. An adaptive deep reinforcement learning framework enables curling robots with human-like performance in real-world conditions. Science Robotics 5, 46 (2020). https://doi.org/10.1126/scirobotics.abb 9764 arXiv:https://robotics.sciencemag.org/content/5/46/eabb9764.full.pdf

Erwin Wu and Hideki Koike. 2020. FuturePong: Real-Time Table Tennis Trajectory Forecasting Using Pose Prediction Network. In Extended Abstracts of the $2020 \mathrm{CHI}$ Conference on Human Factors in Computing Systems (Honolulu, HI, USA) (CHI EA '20). Association for Computing Machinery, New York, NY, USA, 1-8. https: //doi.org/10.1145/3334480.3382853 